\title{
On Portfolio Choice with Savoring and Disappointment
}

\author{
Elyès Jouini, Paul Karehnke and Clotilde Napp*
}

June 12, 2013

\begin{abstract}
We revisit the model proposed by Gollier and Muermann (see Gollier, C. and A. Muermann, 2010, Optimal choice and beliefs with exante savoring and ex-post disappointment, Management Sci., 56, 1272 1284, hereafter GM). In GM, for a given lottery, agents form anticipated expected payoffs and the set of possible anticipations is assumed to be exogenously fixed. We rather propose sets of possible anticipations which are endogenously determined. This permits to compare and evaluate in a consistent manner lotteries with different supports and to revisit the portfolio choice problem. We obtain new conclusions and interesting insights. Our extended model can rationalize a

\footnotetext{
*Jouini: Ceremade and PSL, Université Paris-Dauphine. E-mail: jouini@ceremade.dauphine.fr. Karehnke: DRM and PSL, Université ParisDauphine and Department of Finance and CentER, Tilburg University. E-mail: paul.karehnke@dauphine.fr. Napp: CNRS, DRM and PSL, Université Paris-Dauphine. E-mail: clotilde.napp@dauphine.fr. We thank Rakesh Sarin, an anonymous associate editor and three anonymous referees for their constructive comments and suggestions. We also thank Milo Bianchi as well as participants of the workshop on "Risk preferences and Decisions under Risk" in Berlin and seminar participants at Université Paris-Dauphine for their helpful comments and discussions. The financial support of the GIP-ANR (Risk project) and of the Risk Foundation (Groupama Chair) is gratefully acknowledged by the authors.
} 
variety of empirically observed puzzles like a positive demand for assets with negative expected returns, preference for skewed returns and under-diversification of portfolios.

Keywords: endogenous beliefs; anticipatory feelings; disappointment; optimism; portfolio choice; skewness; under-diversification.

\section{Introduction}

Gollier and Muermann [2010] (hereafter GM) propose a structural model of subjective belief formation in which beliefs solve a trade-off between ex-ante savoring and ex-post disappointment. Models of subjective beliefs (with possible cognitive dissonance) go back to Akerlof and Dickens [1982]. The GM model is in line with this literature and more precisely builds on the optimal beliefs approach introduced by Brunnermeier and Parker [2005] and Brunnermeier et al. [2007], in which the agents form beliefs endogenously and derive ex-ante felicity from expectations of future pleasures; with such an approach, optimal beliefs balance the benefits of higher expectations against the costs of worse decision making and are necessarily biased towards optimism. GM model also builds on the disappointment theory, introduced by Bell [1985], Loomes and Sugden [1986] and Gul [1991], for which the felicity associated to a given uncertain outcome increases with the difference between the realization and the expectation. In GM model, agents form an anticipated expected payoff and optimal beliefs realize the best trade-off between ex-ante savoring and ex-post disappointment: high expectations lead to more ex-ante savoring at the cost of being disappointed ex-post while low expectations lead to the benefits of elation ex-post at the cost of less savoring ex-ante. Depending on the relative weight of the ex-ante and ex-post criteria, the optimal belief might be optimistic or pessimistic, leading to a quite realistic framework to model decision making and to think about endogenous heterogeneous beliefs. 
In this paper, we revisit GM model. In GM the set of possible anticipations is exogenously fixed; we rather propose to relate the set of possible anticipations to the lottery characteristics. The main differences are the following. First, in our setting, as in Brunnermeier and Parker [2005], "in order to believe that something is possible, then it must be possible": feasible subjective probability distributions are assumed to be absolutely continuous with respect to the objective one. In our model, the only possible anticipated expected payoff is the sure payoff when there is no uncertainty: if I get 100 for sure, then I can only believe that I will get 100 . In the case of a lottery yielding 0 or 100 with equal probabilities, an agent can believe that he will win 0 or that he will win 100 or that he will win on average any value between 0 and 100 reflecting a subjective belief that 0 will occur with some probability $p$ and 100 with $1-p$. However, he cannot believe that he will win some value outside $[0,100]$. Second, the welfare level of a given lottery does not depend on the set of 0-probability possible outcomes that can be added to the lottery support. Third and as a consequence, as far as the portfolio choice problem is concerned, the set of possible anticipated expected payoffs is not constrained by exogenous bounds as in GM, but depends upon the level of investment in the risky asset, which seems more natural, since this level modifies the support of the possible payoffs.

Our extended model leads to new conclusions and interesting insights, which shed light on a variety of puzzles in decision theory and in portfolio choice literature.

First, it appears that the preference functional is not necessarily compatible with first-degree and second-degree stochastic dominance. The intuition for this result is that an increase in risk may enlarge the support and may enable the agent to form an optimal anticipated expected payoff which is more favorable in terms of the savoring and disappointment trade-off and thereby may lead to a higher welfare. We provide and discuss an additional 
condition on the preference functional to restore compatibility with firstdegree stochastic dominance: the weight on savoring must be large enough with respect to the weight on disappointment. This condition is consistent with Gneezy et al. [2006], who underline that pure disappointment models permit violations of FSD.

As a consequence, it may be optimal to invest in a risky asset with an expected excess return equal to zero. In our revisited model, risk taking may be optimal even if the expected payoff is negative. The rationale is that investing in the risky asset enables the individual to have a larger range of possible anticipated expected payoffs and possibly a higher welfare.

Third, the agents exhibit preference for skewed returns as in Brunnermeier et al. [2007]: a positive demand for a skewed asset enables the agent to savor more for a given level of risk than the opposite demand. The last two results may explain the popularity of lottery games (Thaler and Ziemba, 1988) despite their negative expected returns and the underperformance of lottery-type stocks (Kumar, 2009, Bali et al., 2011): gambling enables to dream. This taste for lottery-type stocks and for extreme values is also a possible explanation for portfolio under-diversification (Mitton and Vorkink, 2007).

Fourth, the allocation in the risky asset may increase with the weight on savoring, i.e. with the intensity of anticipatory feelings, while in GM, the constant bounds assumption had the implication that the more the agent savors the less risk he takes. GM showed that a larger weight on savoring increases risk aversion and hence reduces the allocation in the risky asset. In our revisited model, we have in addition a support effect which may outweigh the effect of the increase in risk aversion.

Finally, we argue that our revisited model provides a suitable framework to think of simultaneous demand for insurance and lotteries, a puzzle pointed out by Friedman and Savage [1948]. Consistent with Lopes [1987] theory of 
hope and fear and Shefrin and Statman [2000] behavioral portfolio theory, our model can explain the coexistence of insurance and lottery demand with the fear of disappointment and the desire to savor.

In the next section, we present the model, then in Section 3 we analyze its properties. Proofs are provided in the Appendix.

\section{The Model}

We first present our model, that is directly derived from GM, then analyze its relevance and detail the differences with the original model.

\subsection{Our decision criterion and its application to portfolio choice}

The agent faces a risky payoff $\tilde{c}$, described by its (objective) probability distribution $Q$ over the real line. The agent can extract, at date 0 , satisfaction from anticipatory feelings. As in Brunnermeier and Parker [2005], the agent can choose a subjective probability distribution in the set $\mathcal{P}$ of all probability distributions that are absolutely continuous with respect to $Q$. The agent then enjoys at date 0 the subjectively expected future utility of the risky payoff $\tilde{c}$. This satisfaction from anticipatory feelings comes at the cost of experiencing, at date 1, disappointment. Disappointment is measured with respect to a reference point $y$, that we will call the anticipated expected payoff. For a given realization $c$ of $\tilde{c}$, the agent enjoys at date 1 the satisfaction $U(c, y)$, where $U$ is a bidimensional utility function increasing and concave in its first argument, i.e., such that $U_{c}>0$ and $U_{c c}<0$ and decreasing in the second argument, i.e., such that $U_{y}<0$ in order to reflect disappointment. The higher the anticipated expected payoff, the higher the ex-post disappointment ${ }^{1}$. The intertemporal welfare of the agent for a

\footnotetext{
${ }^{1}$ As underlined by Caplin and Leahy [2001], "have you ever felt disappointed about an outcome without having experienced prior feelings of hopefulness ?"
} 
given choice of belief $P$ in $\mathcal{P}$ is a weighted sum of his ex-ante and ex-post satisfactions and given by $W(P, \tilde{c})=k E^{P}[U(\tilde{c}, y)]+E^{Q}[U(\tilde{c}, y)]$, where $k$ measures the intensity of anticipatory feelings. The anticipated expected payoff $y$ is defined as the (subjective) certainty equivalent of the risky payoff, i.e., $U(y, y)=E^{P}[U(\tilde{c}, y)]$. We assume that the function $v(y) \equiv U(y, y)$ is increasing in $y$ to reflect the fact that receiving a higher payoff in line with expectations increases the agent's utility ${ }^{2}$. Since $U(y, y)=E^{P}[U(\tilde{c}, y)]$, it also means that increasing the anticipated expected payoff raises at date 0 the satisfaction extracted from anticipatory feelings. Remark that since $W(P, c)=(k+1) U(c, c)$ for a deterministic $c$, the condition on $v$ is also a monotonicity condition on the welfare function over the set of sure payoffs, which is natural.

The agent's optimization problem $\left(\mathcal{O}_{\mathcal{P}}\right)$ consists in selecting a subjective belief $P$ in $\mathcal{P}$ in order to maximize his welfare $W(P, \tilde{c})$. Letting $c_{\inf (Q)}$ and $c_{\sup (Q)}$ denote the essential infimum and essential supremum of $\tilde{c}$ under $Q$, it is easy to get that the agent's optimization problem $\left(\mathcal{O}_{\mathcal{P}}\right)$ is equivalent to the following optimization problem $\left(\mathcal{O}_{y}\right)$

$$
\max _{c_{\inf (Q)} \leq y \leq c_{\sup (Q)}} E^{Q}[F(\tilde{c}, y)],
$$

where $F(c, y)=k U(y, y)+U(c, y)$. The agent is then endowed with a decision criterion, that associates with every risky payoff $\tilde{c}$ a welfare level $W(\tilde{c}) \equiv \max _{c_{\inf (Q)} \leq y \leq c_{\sup (Q)}} E^{Q}[F(\tilde{c}, y)]$, corresponding to the optimal tradeoff between ex-ante savoring and ex-post disappointment. ${ }^{3}$

Note that the optimization problem $\left(\mathcal{O}_{y}\right)$ is also consistent with the (sub-

\footnotetext{
${ }^{2}$ One prefers to consume $\$ 6,000$ in line with expectations rather than $\$ 5,000$ in line with expectations.

${ }^{3}$ Note that we would obtain analogous results if we considered the more general optimization problem $\max _{c_{\inf (Q)} \leq y \leq c_{\sup (Q)}} k v(y)+E^{Q}[U(\tilde{c}, y)]$ for a general increasing function $v$ (i.e. not necessarily of the form $v(y)=U(y, y))$ such that $F(c, y)=k v(y)+U(c, y)$ is concave in $y$. In particular this permits to consider different date 0 and date 1 utility functions.
} 
jective) expected value of the risky payoff as the reference point, instead of the certainty equivalent. Indeed, the optimization problem $\max _{P \in \mathcal{P}} k U\left(E^{P}[\tilde{c}], E^{P}[\tilde{c}]\right)+$ $E^{Q}\left[U\left(\tilde{c}, E^{P}[\tilde{c}]\right)\right]$ is equivalent to the optimization problem $\left(\mathcal{O}_{y}\right) \cdot{ }^{4}$ This means that our model is consistent with models of disappointment that adopt the certainty equivalent as reference point, as in Gul [1991], as well as with models that adopt the expected payoff as the reference point as in Bell [1985] and Loomes and Sugden [1986].

Let us now consider the standard portfolio choice problem with such a decision criterion. The agent has some initial wealth $z$ at date 0 , that can be invested in a riskless asset, whose return between date 0 and date 1 is normalized to one, and in a risky asset, whose excess return is described by a random variable $\widetilde{x}$, with probability distribution $Q$. When the agent invests a level $\alpha$ of his wealth in the risky asset, then he faces the risky payoff $\tilde{c}_{\alpha}=(z+\alpha \tilde{x})$ and, by (1), his intertemporal welfare is given by $W\left(\tilde{c}_{\alpha}\right)=\max _{\left(c_{\alpha}\right)_{\text {inf }} \leq y \leq\left(c_{\alpha}\right)_{\text {sup }}} E^{Q}\left[F\left(\tilde{c}_{\alpha}, y\right)\right]$. The agent's portfolio choice problem then consists in choosing the level $\alpha^{*}$ of wealth invested in the risky asset in order to maximize his intertemporal welfare, i.e. such that $\alpha^{*}=\arg \max _{\alpha} W\left(\tilde{c}_{\alpha}\right)$.

In the remainder of the paper, and as in GM, we make the regularity assumption that the function $F(c, y)$ is concave in $y$. The following firstorder condition is then necessary and sufficient to determine the optimal anticipated expected payoff $y^{*}$

$$
E^{Q}\left[F_{y}(\tilde{c}, y)\right]=k v^{\prime}(y)+E^{Q}\left[U_{y}(\tilde{c}, y)\right] \begin{cases}\leq 0 & \text { if } y^{*}=c_{\inf (Q)}, \\ =0 & \text { if } y^{*} \in\left(c_{\inf (Q)}, c_{\sup (Q)}\right), \\ \geq 0 & \text { if } y^{*}=c_{\sup (Q)} .\end{cases}
$$

We shall repeatedly consider the additive habit formation specification

\footnotetext{
${ }^{4}$ This does not mean that it is possible to replace $y$ with the subjective expected value of the risky payoff in the initial problem of GM.
} 
developed by Constantinides [1990], $U(c, y)=u(c-\eta y)$, for an increasing and concave function $u$ and a positive scalar $\eta<1$. It is easy to verify that this bidimensional function satisfies all the above regularity assumptions.

\subsection{Our model vs. GM model}

Let us be clear about the distinction between the seminal model of GM and our extended model and about the relevance of our modifications. GM fix a finite set of possible payoffs $C=\left\{c_{1}<c_{2}<\ldots<c_{S}\right\}$ and provide a decision criterion for the set $\mathcal{S}_{C}$ of simple lotteries, whose support is in $C$. A lottery $Q$ in $\mathcal{S}_{C}$ is described by a vector of probabilities $\left(q_{1}, q_{2}, \ldots, q_{S}\right)$ with $q_{i} \geq 0$ and $\sum_{i=1}^{S} q_{i}=1$. For any lottery $Q$ in $\mathcal{S}_{C}$, the agent's welfare $W(Q)$ is given by $W(Q)=\max _{c_{1} \leq y \leq c_{S}} k U(y, y)+\sum_{s=1}^{S} q_{s} U\left(c_{s}, y\right)$. The welfare level of $Q$ does not depend upon its support but depends on $C$ (through $c_{1}$ and $c_{S}$ ). Notice the difference with our decision criterion where the bounds are given by $c_{\text {inf }\left\{i ; q_{i}>0\right\}}$ and $c_{\sup \left\{i ; q_{i}>0\right\}}$. In fact, the agent in GM model can choose a subjective probability that is singular with respect to the objective one whereas the agent in our model is constrained to choose a probability that is absolutely continuous with respect to the objective one. As a result, when there is no uncertainty, the only possible (and optimal) anticipated expected payoff is equal to the sure payoff in our model. We think that this feature is reasonable since if there is no uncertainty, then there is nothing to dream or to be disappointed about. In GM model the optimal anticipated expected payoff is also equal to the sure payoff only if $F_{y}(x, x)=0$ for all $x$, or if the set of possible payoffs is reduced to a singleton, namely the sure payoff. For the additive habit specification, $F_{y}(x, x)=0$ for all $x$ is satisfied only if $k=\frac{\eta}{1-\eta}$. More generally, in our setting, the anticipated expected payoff belongs to the (convex hull of the) support of the objective lottery.

Note that in the case where the support of the objective distribution of the lottery $Q$ under consideration coincides with the set $C$ of possible payoffs 
in GM, then $c_{1}=c_{\inf \left\{i ; q_{i}>0\right\}}$ and $c_{S}=c_{\sup \left\{i ; q_{i}>0\right\}}$, and the welfare level of $Q$ in GM coincides with its welfare level in our extended model. But then GM model only permits to compare lotteries with the same support. Our decision criterion can then be seen as an extension of GM decision criterion to lotteries with different supports. In the case where the set $C$ in GM and the support of the objective distribution do not coincide, the model presented here is not exactly an extension but rather a modification of GM, since it does not lead to the same welfare levels.

As far as the portfolio choice problem is concerned, GM impose exogenous bounds $y_{\text {inf }}$ and $y_{\text {sup }}$ on anticipated expected payoffs and these bounds are the same for all payoffs $\tilde{c}_{\alpha}=z+\alpha \tilde{x}$, independently of $\alpha$. In our model, if an agent does not invest in the risky asset $(\alpha=0)$, the only possible (and optimal) anticipated expected payoff is equal to the sure payoff $z\left(y^{*}(0)=z\right)$ : the individual cannot extract anticipatory feelings without investing in the risky asset. In GM, the agent can choose any anticipated expected payoff in $\left[y_{\text {inf }}, y_{\text {sup }}\right]$, even though he is sure to get $z$ : the individual can savor a high anticipated expected payoff even if he does not invest in the risky asset, and is hence sure to keep the same wealth $z$. More generally, in our model, the level of investment modifies the range of possible realizations hence of possible anticipated expected payoffs.

\section{$3 \quad$ Results and Predictions}

Our extended model leads to new conclusions and interesting insights.

\subsection{Comparative statics}

\subsubsection{Optimal anticipated expected payoffs.}

First, it is easy to show, exactly as in GM, that an increase in the intensity of anticipatory feelings weakly increases the optimal anticipated expected 
payoff, i.e. $\frac{\partial y^{*}}{\partial k} \geq 0$. As intuition suggests, when the intensity of anticipatory feelings increases, the agent can get more benefits from his dreams and biases his beliefs towards more optimism.

Most results in GM about the impact of stochastic dominance on the optimal anticipated expected payoff are not valid anymore in our setting without additional assumptions. Detailed stylized counterexamples can be found in Appendix A, but the main idea is the following: in our extended model, modifying the support of the objective distribution changes the range of the possible anticipated expected payoffs, and may authorize anticipated expected payoffs which are more favorable in terms of the savoring and disappointment trade-off. The only result that remains valid is the following.

Proposition 3.1. If $U_{y}$ is increasing in the payoff $c$, then any FSD dominated shift in the probability distribution $Q$ weakly reduces the optimal anticipated expected payoff $y^{*}$.

The condition $U_{y c}>0$ means that the agent is disappointment averse. Notice that for the habit formation specification $U(c, y)=u(c-\eta y)$, we always have $U_{y c}>0$.

\subsubsection{Welfare.}

GM show that any SSD dominated shift (and in particular, any FSD dominated shift) in the probability distribution $Q$ weakly reduces the agent's intertemporal welfare (Proposition 5). In our setting, in the absence of additional condition, the impact of a FSD dominated shift on welfare is ambiguous $^{5}$. As just seen, modifying the support of the objective distribution may authorize more favorable trade-offs between savoring and disappointment, and then lead to higher welfare.

\footnotetext{
${ }^{5}$ An example of a FSD dominated shift leading to a decrease in welfare can be found in Appendix A (Example 4).
} 
The simplest forms of FSD dominated shifts are given by the shift from the binary lottery $L=\left(\left\{x_{1}, x_{2}\right\},(\pi, 1-\pi)\right)$ with $x_{1}<x_{2}$ to the sure payoff $x_{1}$ or by the shift from the sure payoff $x_{2}$ to the lottery $L$. Gneezy et al. [2006] define as the internality axiom for decision models the fact that for any binary lottery these two simple shifts reduce welfare. Equivalently, this axiom imposes that for any binary lottery, the welfare level associated to the lottery ranges between the welfare level of its lowest and highest outcomes. Note that, as underlined by Gneezy et al. [2006], disappointment models permit violations of the internality requirement. This means that even with this simplest form of FSD, an additional condition is needed for our decision criterion. The following result shows that the internality requirement is equivalent to the condition $F_{y}(x, x) \geq 0$ for all $x$. Moreover, it also shows that this condition guarantees that our decision criterion is consistent with FSD shifts.

Proposition 3.2. The three following conditions are equivalent:

1. The decision criterion $W$ satisfies the internality requirement.

2. For all $x, F_{y}(x, x) \geq 0$.

3. Any FSD dominated shift in the probability distribution $Q$ weakly reduces the agent's intertemporal welfare.

The condition $F_{y}(x, x) \geq 0$ for all $x$ is a condition on the relative weights of savoring and disappointment. It amounts to assuming that when the anticipated expected payoff and the payoff are in line, the decrease in exante utility induced by a decrease in the anticipated expected payoff - due to lower anticipatory feelings - is greater than the increase in ex-post utility due to lower disappointment. A slight decrease ${ }^{6}$ in the anticipated expected

\footnotetext{
${ }^{6}$ This local property is also satisfied at the global level and slight decreases might be replaced by general decreases. Indeed, since $F$ is concave in $y$, the condition $F_{y}(x, x) \geq 0$
} 
payoff then induces a decrease in intertemporal welfare. Since, as underlined above, pure models of disappointment violate the internality requirement, our condition ensures that the weight on savoring is high enough compared to the weight on disappointment to induce the agent to bias his beliefs upwards, when the anticipated expected payoff and the actual payoff are in line. For the habit formation specification $U(c, y)=u(c-\eta y)$, the additional condition $F_{y}(x, x) \geq 0$ for all $x$ is satisfied if and only if $k \geq \frac{\eta}{1-\eta}$.

Finally, we show in Table 1 in the Appendix that for some specifications, our model, as GM model, can help explain Allais paradox.

\subsection{Positive demand for assets with negative expected return}

The following proposition shows that the agent may take nonzero positions on zero mean risk assets in contrast with Proposition 8 of GM and in contrast with the standard expected utility model. As previously, the intuition is the following: in our setting, the presence of risk permits a larger range of possible anticipated expected payoffs hence possibly higher savoring or less disappointment compensating for risk aversion.

Proposition 3.3. Let $\tilde{x}$ be a bounded, nonzero, zero-mean risk and let $z$ denote the agent's initial wealth. If $F_{y}(z, z) \neq 0$, then the optimal investment $\alpha^{*}$ in the risky asset $\tilde{x}$ is nonzero.

This proposition shows that there are zero mean risks for which the optimal demand is positive (even if it means changing $\tilde{x}$ into $-\tilde{x}$ ). Slight perturbations of $\tilde{x}$ or $-\tilde{x}$ would then permit to construct negative mean risks for which the optimal demand is positive. Note that State lotteries typically have a negative average payoff. In our framework, the positive demand for such lotteries is rationalized by the savoring of favorable future prospects. Given the equivalence between portfolio choice and insurance for all $x$ is equivalent to the fact that the function $y \mapsto F(x, y)$ is nondecreasing on $\{y \leq x\}$. 
demand problems, Proposition 3.3 also shows that full insurance is not optimal for actuarially fair insurance when $F_{y}(z, z) \neq 0$ which may help to explain the annuities puzzle ${ }^{7}$. More generally, the proposition implies that risky prospects might be desirable. This explains why there is no systematic effect of SSD shifts on welfare in our setting (see Example 3, Appendix A).

For the habit formation specification $U(c, y)=u(c-\eta y)$, we have $F_{y}(z, z) \neq 0$ for all $z$ if and only if $k \neq \frac{\eta}{1-\eta}$. Under this assumption, Proposition 3.3 applies for all possible initial wealth levels, and the agent might then invest in a risky asset with a negative expected return. For example, for $k>\frac{\eta}{1-\eta}$ and $E^{Q}[\tilde{x}]<0$, we can see, using the proof of Proposition 3.3, that, if shortsales are not allowed, the optimal investment level $\alpha^{*}$ is positive as soon as $x_{\text {sup }}>-\frac{E^{Q}[\tilde{x}]}{k(1-\eta)-\eta}$ or in other words, as soon as the expected loss is moderate relative to the maximum possible gain. This is typically the case with State lotteries for which the expected gain is negative, shortsales are not allowed and the maximum possible gain is high. Note that the focus on the maximum possible gain is consistent with Cook and Clotfelter [1993], who document that per capita lottery sales increase with the population base: indeed a higher possible jackpot makes higher dreams possible.

It is also interesting to note that under the condition $F_{y}(x, x) \geq 0$, SSD dominated shifts are undesirable when they do not affect the maximum possible dream.

Proposition 3.4. Assume that $F_{y}(x, x) \geq 0$ for all $x$. A SSD dominated shift in the probability distribution $Q$ which does not modify the maximum possible payoff weakly reduces the agent's intertemporal welfare.

This result might help to explain simultaneous demand for insurance and lotteries: an agent who holds a lottery ticket and faces some risk which

\footnotetext{
${ }^{7}$ See for instance Benartzti et al. [2011] for a review on this subject
} 
does not affect the lottery jackpot would be interested by a risk reduction through insurance.

\subsection{Under-diversification}

An interesting corollary of Proposition 3.3 is the possible preference for under-diversified portfolios. Indeed, let us consider a financial market with several assets and with a zero idiosyncratic risk. A perfectly diversified portfolio would then be non-risky. Let us normalize its return to zero. Proposition 3.3 implies that when facing the perfectly diversified portfolio and any other under-diversified portfolio with zero average return, an agent with a total wealth $z$ and such that $F_{y}(z, z) \neq 0$ would choose to invest a nonzero fraction of his wealth in the under-diversified portfolio leading to an underdiversified overall portfolio, while a classical expected utility agent would choose to invest his whole wealth in the perfectly diversified portfolio.

Under-diversified portfolio holdings of individual investors have been documented for instance by Mitton and Vorkink [2007] and Goetzmann and Kumar [2008]; they find that under-diversified portfolio holdings are concentrated in stocks with high idiosyncratic volatility and high skewness, i.e. stocks with maximum upside potential. This is consistent with our model that predicts that agents under-diversify in order to savor the upside potential.

\subsection{Binary risk and preference for skewed returns}

In this section, we assume that $U(c, y)=\ln (c-\eta y)$ and that $\tilde{x}$ is a binary risk.

The next proposition solves the portfolio choice problem for general zeromean binary risks and shows a preference for skewed returns. This is consistent with, e.g. Mitton and Vorkink [2007], who find that "investors sacrifice mean variance efficiency for higher skewness exposure". 
Proposition 3.5. Let $z$ denote the agent's initial wealth. Suppose that $U(c, y)=\ln (c-\eta y)$ for $0<\eta<1$, and that the excess return of the risky asset has a zero mean and yields $x_{\mathrm{sup}}>0$ with probability $\pi$ and $x_{\mathrm{inf}}<0$ with probability $1-\pi$. For $\pi \leq \frac{1}{2}$ (resp. $\pi \geq \frac{1}{2}$ ), the optimal investment level is given by $\alpha^{*} \equiv \alpha_{1}=\frac{k(1-\eta)-\eta}{(k+1)\left(\eta x_{\mathrm{sup}}-x_{\mathrm{inf}}\right)} z \quad\left(\right.$ resp. $\left.\alpha^{*} \equiv \alpha_{2}=-\frac{k(1-\eta)-\eta}{(k+1)\left(x_{\mathrm{sup}}-\eta x_{\mathrm{inf}}\right)} z\right)$, with $y\left(\alpha_{1}\right)=\frac{k+\pi}{(k+1)(\pi+\eta(1-\pi))} z$ (resp. $\left.y\left(\alpha_{2}\right)=\frac{k+1-\pi}{(k+1)(1-\pi+\eta \pi)} z\right)$.

In particular, it is optimal not to invest in the zero mean return portfolio, i.e. $\alpha^{*}=0$, if and only if $k=\frac{\eta}{1-\eta}$; in this case, $y^{*}=z$. In the general case, the optimal investment is nonzero. Note that we only need to consider one of the two cases $\pi \leq \frac{1}{2}$ or $\pi \geq \frac{1}{2}$ since they are symmetric.

The case $\pi \leq \frac{1}{2}$ corresponds to a positively skewed distribution of payoffs, hence to a positively skewed range of values for the anticipated expected payoff. When the intensity of anticipatory feelings is high enough relative to the intensity of disappointment $\left(k>\frac{\eta}{1-\eta}\right)$, then the positive skewness enables the agent to dream. In order to savor these high possible anticipated expected payoffs at date 0 , the agent has a positive optimal demand $\alpha^{*}=$ $\alpha_{1}=\frac{k(1-\eta)-\eta}{(k+1)\left(\eta x_{\mathrm{sup}}-x_{\mathrm{inf}}\right)} z>0$ and an optimistic optimal subjective belief $y^{*}=$ $y\left(\alpha_{1}\right)=\left(c_{\alpha_{1}}\right)_{\text {sup }}=z+\alpha_{1} x_{\text {sup }}>z$. When the intensity of disappointment is high enough relative to the intensity of anticipatory feelings $\left(\frac{\eta}{1-\eta}>k\right)$, then the negative skewness of the random variable $(-\tilde{x})$ enables the agent to profit from elation. The agent has then a negative demand of $\tilde{x}$ (or equivalently a positive demand of the negatively skewed risky payoff $-\tilde{x}$ ) with $\alpha^{*}=\alpha_{1}=\frac{k(1-\eta)-\eta}{(k+1)\left(\eta x_{\mathrm{sup}}-x_{\mathrm{inf}}\right)} z<0$ and a pessimistic optimal belief $y^{*}=y\left(\alpha_{1}\right)=z+\alpha_{1} x_{\text {sup }}<z$ in order to benefit from elation at date 1 . We retrieve the fact that depending on the relative intensity of anticipatory feelings and disappointment, the agent's optimal belief can be pessimistic or optimistic.

Moreover, it is easy to get that $\frac{\partial \alpha_{1}}{\partial k}>0$ and $\frac{\partial \alpha_{1}}{\partial \eta}<0$, which means that the optimal investment in a positively skewed asset increases with $k$ and 
decreases with $\eta$. As intuition suggests, a higher intensity of anticipatory feelings, which, as seen in Section 3.1 is associated with more optimism, leads to a higher position in a positively skewed risky asset and a higher intensity of disappointment reduces the level of investment in the positively skewed risky asset. Here again, the implications of our model differ from those of GM's model, since GM find that, for the additive habit specification with $u$ DARA, the optimal investment in the risky asset decreases with $k$ (Proposition 9.1), and that the optimal investment in the risky asset decreases with (resp. increases with, is independent of) $\eta$ iif relative risk aversion is larger than (smaller than, equal to) 1 (Proposition 9.2).

Figure 1 in the Appendix illustrates Proposition 3.5 for $k>\eta /(1-\eta)$, i.e. when the intensity of anticipatory feelings is high enough relative to the intensity of disappointment. The top graph represents the welfare $W(\alpha)$ as a function of the investment in a symmetric binary risk asset. Since the risk is symmetric, there are two symmetric possible values for the optimal portfolio $\alpha^{*}$ yielding the same welfare. Note that the welfare function is not globally concave in $\alpha$. When the return is positively skewed (second graph), the welfare still has two local maxima but only the positive one is a global maximum. The positive demand for the risky asset yields higher welfare because the maximum return $x_{\text {sup }}$ is higher (in absolute value) than the minimum return $x_{\text {inf }}$. Therefore, a positive demand for the asset enables the agent to savor more for a given level of risk than the opposite demand. The third graph represents the symmetric situation with negatively skewed returns.

Figure 2 in the Appendix represents $W(\alpha)$ and illustrates the impact of $k$ in a symmetric returns framework. For $k=1$ (which corresponds, in the example, to $\frac{\eta}{1-\eta}$ ) the optimal demand is zero. When $k$ increases, zero becomes a local minimum of the welfare function and the two symmetric maxima go away from zero. 


\section{A Stylized counterexamples for comparative stat- ics results}

The following examples illustrate the differences between our model and GM model in terms of comparative statics. Their stylized feature permits to clearly highlight the differences.

\section{Example 1: FSD and the optimal anticipated expected payoff.}

$A$ utility function such that $U_{c y}<0$ for which there is a FSD dominated shift that decreases the optimal anticipated expected payoff $y^{*}$.

Let $U$ be defined by $U(c, y)=c-\eta y-\frac{1}{2} \beta(c+\eta y)^{2}$ on $[0,1] \times[0,1]$ with $\beta=\frac{4}{19}$ and $\eta=\frac{1}{2}$. We take $k=2, Q^{1}\left(\left\{\frac{1}{2}\right\}\right)=Q^{1}(\{1\})=\frac{1}{2}$ and $Q^{2}(\{0\})=Q^{2}\left(\left\{\frac{1}{2}\right\}\right)=\frac{1}{2}$. We have $Q^{1} \succ_{F S D} Q^{2}$ and $y^{2}=y^{*}\left(Q^{2}\right)=$ $\frac{1}{2}-\frac{1}{38}<\frac{1}{2}=y^{*}\left(Q^{1}\right)=y^{1}$.

Example 2: Increases in risk and the optimal anticipated expected payoff.

2a. A utility function such that $U_{c c y}<0$ and for which there is an increase in risk in the sense of Rothschild-Stiglitz that increases the optimal anticipated expected payoff $y^{*}$.

Let $U$ be defined by $U(c, y)=\ln \left(c-\frac{1}{2} y\right)$ on $\left[\frac{9}{10}, \frac{11}{10}\right] \times\left[\frac{9}{10}, \frac{11}{10}\right]$. We take $k=3, Q^{1}$ and $Q^{2}$ such that $Q^{1}(\{1\})=1$ and $Q^{2}\left(\left\{\frac{9}{10}\right\}\right)=Q^{2}\left(\left\{\frac{11}{10}\right\}\right)=\frac{1}{2}$. The distribution $Q^{2}$ is more risky than $Q^{1}$ in the sense of Rothschild-Stiglitz and we have $y^{2}=y^{*}\left(Q^{2}\right)=\frac{11}{10}>1=y^{*}\left(Q^{1}\right)=y^{1}$.

2b. A utility function such that $U_{c c y}>0$ and for which there exists an increase in risk in the sense of Rothschild-Stiglitz that decreases the optimal anticipated expected payoff $y^{*}$.

Let $U$ be defined by $U(c, y)=c-\frac{1}{2} y-\frac{1}{4}\left(c-\frac{1}{2} y\right)^{2}+\frac{1}{86}\left(c+\frac{1}{2} y\right)^{3}$ on $\left[\frac{9}{10}, \frac{11}{10}\right] \times$ $\left[\frac{9}{10}, \frac{11}{10}\right]$. We take $k=\frac{1}{2}, Q^{1}(\{1\})=1$ and $Q^{2}\left(\left\{\frac{9}{10}\right\}\right)=Q^{2}\left(\left\{\frac{11}{10}\right\}\right)=\frac{1}{2}$. 
The distribution $Q^{2}$ is more risky than $Q^{1}$ in the sense of Rothschild-Stiglitz and we have $y^{2}=y^{*}\left(Q^{2}\right)=\frac{9}{10}<1=y^{*}\left(Q^{1}\right)=y^{1}$.

\section{Example 3: SSD and welfare.}

A SSD dominated shift in the probability distribution $Q$ that increases the intertemporal welfare.

Take the same utility function and the same distributions as in $2 \mathrm{a}$. We check that $W\left(Q^{1}\right)<W\left(Q^{2}\right)$.

\section{Example 4: FSD and welfare.}

A FSD dominated shift in the probability distribution $Q$ that increases intertemporal welfare.

Let $U$ be defined by $U(c, y)=\ln \left(c-\frac{1}{2} y\right)$ on $\left[\frac{9}{10}, 1\right] \times\left[\frac{9}{10}, 1\right]$. We take $k=$ $\frac{1}{2}, Q^{1}(\{1\})=1$ and $Q^{2}\left(\left\{\frac{9}{10}\right\}\right)=1-Q^{2}(\{1\})=0.01$. We have $Q^{1} \succeq_{F S D} Q^{2}$ and we check that $W\left(Q^{1}\right)<W\left(Q^{2}\right)$.

\section{B Proofs}

\section{Proof of Proposition 3.1.}

Let $Q^{1} \succeq_{F S D} Q^{2}$. For $i=1,2$, we denote by $y^{i}, c_{\text {inf }}^{Q^{i}}$ and $c_{\text {sup }}^{Q^{i}}$ the optimal anticipated expected payoff, the essential infimum and the essential supremum under $Q^{i}$. Since $U_{c y}>0$, we have $F_{c y}>0$ and then $E^{Q^{1}}\left[F_{y}\left(\widetilde{c}, y^{1}\right)\right] \geq E^{Q^{2}}\left[F_{y}\left(\widetilde{c}, y^{1}\right)\right]$. Furthermore, FSD shifts the support to lower payoffs that is, $c_{\mathrm{sup}}^{Q^{1}} \geq c_{\mathrm{sup}}^{Q^{2}}$ and $c_{\mathrm{inf}}^{Q^{1}} \geq c_{\mathrm{inf}}^{Q^{2}}$. The domain over which $E^{Q^{2}}\left[F_{y}(\widetilde{c}, y)\right]$ is maximized intersects then $\left(-\infty, y^{1}\right]$. If $E^{Q^{1}}\left[F_{y}\left(\widetilde{c}, y^{1}\right)\right] \leq 0$, then $E^{Q^{2}}\left[F_{y}\left(\widetilde{c}, y^{1}\right)\right] \leq 0$ and since $F$ is concave in $y$, we have $y^{2} \leq y^{1}$. If $E^{Q^{1}}\left[F_{y}\left(\widetilde{c}, y^{1}\right)\right]>0$, then $y^{1}$ corresponds to the highest possible payoff under $Q^{1}$ and we necessarily have $y^{2} \leq y^{1}$. 


\section{Proof of Proposition 3.2.}

$(2) \Rightarrow(1)$ : Consider the lottery $L=\left(\left\{x_{1}, x_{2}\right\},(\pi, 1-\pi)\right)$ with $x_{1}<x_{2}$ and denote by $y^{*}$ the optimal anticipated expected payoff of the lottery. We have $W\left(x_{1}\right)=F\left(x_{1}, x_{1}\right) \leq \pi F\left(x_{1}, x_{1}\right)+(1-\pi) F\left(x_{2}, x_{1}\right) \leq \pi F\left(x_{1}, y^{*}\right)+$ $(1-\pi) F\left(x_{2}, y^{*}\right)=W(L)$, where the first inequality is due to $F_{c}>0$ and the second inequality comes from the optimality of $y^{*}$. The inequality $W\left(x_{1}\right) \leq$ $W(L)$ is then always satisfied.

Since $F_{y y}<0, F_{y}\left(x_{2}, x_{2}\right) \geq 0$ implies that $F_{y}\left(x_{2}, x\right) \geq F_{y}\left(x_{2}, x_{2}\right) \geq 0$ for $x \leq x_{2}$ and then $F\left(x_{2}, x\right) \leq F\left(x_{2}, x_{2}\right)$ for all $x \leq x_{2}$. Thus, we have $W(L)=\pi F\left(x_{1}, y^{*}\right)+(1-\pi) F\left(x_{2}, y^{*}\right) \leq F\left(x_{2}, y^{*}\right) \leq F\left(x_{2}, x_{2}\right)=W\left(x_{2}\right)$, where the first inequality follows from $F_{c}>0$.

$(1) \Rightarrow(2)$ : Assume that there exist $x_{2}$ and $y<x_{2}$ with $F\left(x_{2}, y\right)>$ $F\left(x_{2}, x_{2}\right)$. Let $x_{1}<y$ and consider the lottery $l=\left(\left\{x_{1}, x_{2}\right\},(\pi, 1-\pi)\right)$ with optimal anticipated expected payoff denoted by $y^{l}$. We have $W(l)=$ $\pi F\left(x_{1}, y^{l}\right)+(1-\pi) F\left(x_{2}, y^{l}\right)$, hence by optimality, $W(l) \geq \pi F\left(x_{1}, y\right)+(1-$ $\pi) F\left(x_{2}, y\right)$. Choosing $\pi$ small enough, we have $W(l)>F\left(x_{2}, x_{2}\right)=W\left(x_{2}\right)$, which leads to a contradiction. For all $x_{2}$, we then have $F\left(x_{2}, y\right) \leq F\left(x_{2}, x_{2}\right)$ for all $y \leq x_{2}$, hence $F_{y}\left(x_{2}, x_{2}\right) \geq 0$.

$(2) \Rightarrow(3)$ : Let $Q^{1} \succeq_{F S D} Q^{2}$ and let $y^{1}$ and $y^{2}$ denote the optimal anticipated expected payoffs respectively associated to $Q^{1}$ and $Q^{2}$. Since $F_{c}>0$, we have $W\left(Q^{2}\right)=E^{Q^{2}}\left[F\left(\widetilde{c}, y^{2}\right)\right] \leq E^{Q^{1}}\left[F\left(\widetilde{c}, y^{2}\right)\right]$. If $y^{2} \geq c_{\mathrm{inf}}^{Q^{1}}$ then $y^{2} \in\left[c_{\mathrm{inf}}^{Q^{1}}, c_{\mathrm{sup}}^{Q^{1}}\right]$ (see the proof of Proposition 3.1) and, by optimality, we have $E^{Q^{1}}\left[F\left(\widetilde{c}, y^{2}\right)\right] \leq E^{Q^{1}}\left[F\left(\widetilde{c}, y^{1}\right)\right]=W\left(Q^{1}\right)$. If $y^{2}<c_{\text {inf }}^{Q^{1}}$, we have, for all $c$ in the support of $Q^{1}, F\left(c, y^{2}\right) \leq F\left(c, c_{\text {inf }}^{Q^{1}}\right)$ since $F_{y}(x, x) \geq 0$ for all $x$ implies that $F(c, y)$ is increasing in $y$ for $y \leq c$ (see above). Therefore, $E^{Q^{1}}\left[F\left(\widetilde{c}, y^{2}\right)\right] \leq E^{Q^{1}}\left[F\left(\widetilde{c}, c_{\text {inf }}^{Q^{1}}\right)\right] \leq E^{Q^{1}}\left[F\left(\widetilde{c}, y^{1}\right)\right]=W\left(Q^{1}\right)$, where the last inequality is due to the optimality of $y^{1}$.

$(3) \Rightarrow(1)$ : immediate. 


\section{Proof of Proposition 3.3.}

Assume that $F_{y}(z, z)>0$. For $\alpha>0$ and sufficiently small, $y^{*}(\alpha)$ is sufficiently close to $z$ to have $k v^{\prime}\left(y^{*}(\alpha)\right)+E^{Q}\left[U_{y}\left(\tilde{c}_{\alpha}, y^{*}(\alpha)\right]>0\right.$. This implies that $y^{*}(\alpha)=\left(c_{\alpha}\right)_{\text {sup }}$. Hence, for $\alpha>0$ sufficiently small, $W_{\alpha}(\alpha)=$ $E\left[\tilde{x} U_{c}\left(\tilde{c}_{\alpha}, y^{*}(\alpha)\right)+x_{\text {sup }} F_{y}\left(\tilde{c}_{\alpha}, y^{*}(\alpha)\right)\right]$ and $\lim _{\alpha \rightarrow 0^{+}} W_{\alpha}(\alpha)=x_{\text {sup }} F_{y}(z, z)>$ 0 . We prove similarly that $\lim _{\alpha \rightarrow 0^{-}} W_{\alpha}(\alpha)=x_{\text {inf }} F_{y}(z, z)<0$ and $\alpha=0$ is a local minimum for $W(\alpha)$. The optimal investment is then nonzero. The case $F_{y}(z, z)<0$ is treated similarly.

\section{Proof of Proposition 3.4}

Let $Q^{1} \succeq_{S S D} Q^{2}$ with $c_{\text {sup }}^{Q^{1}}=c_{\text {sup }}^{Q^{2}}$ and let $y^{1}$ and $y^{2}$ denote the optimal anticipated expected payoffs respectively associated to $Q^{1}$ and $Q^{2}$. Since $F$ is concave in $c$, we have $W\left(Q^{2}\right)=E^{Q^{2}}\left[F\left(\widetilde{c}, y^{2}\right)\right] \leq E^{Q^{1}}\left[F\left(\widetilde{c}, y^{2}\right)\right]$. If $y^{2} \geq c_{\mathrm{inf}}^{Q^{1}}$ then $y^{2} \in\left[c_{\mathrm{inf}}^{Q^{1}}, c_{\mathrm{sup}}^{Q^{1}}\right]$ (since by assumption $c_{\mathrm{sup}}^{Q^{1}}=c_{\mathrm{sup}}^{Q^{2}}$ ) and, by optimality, we have $E^{Q^{1}}\left[F\left(\widetilde{c}, y^{2}\right)\right] \leq E^{Q^{1}}\left[F\left(\widetilde{c}, y^{1}\right)\right]=W\left(Q^{1}\right)$. If $y^{2}<c_{\text {inf }}^{Q^{1}}$, we have, for all $c$ in the support of $Q^{1}, F\left(c, y^{2}\right) \leq F\left(c, c_{\text {inf }}^{Q^{1}}\right)$ since $F_{y}(x, x) \geq$ 0 for all $x$ implies that $F(c, y)$ is increasing in $y$ for $y \leq c$. Therefore, $E^{Q^{1}}\left[F\left(\widetilde{c}, y^{2}\right)\right] \leq E^{Q^{1}}\left[F\left(\widetilde{c}, c_{\text {inf }}^{Q^{1}}\right)\right] \leq E^{Q^{1}}\left[F\left(\widetilde{c}, y^{1}\right)\right]=W\left(Q^{1}\right)$, where the last inequality is due to the optimality of $y^{1}$.

\section{Proof of Proposition 3.5.}

Let us first study the concavity of $W$ and let us assume $\alpha>0$ (the case $\alpha<0$ is treated similarly).

If $\left(c_{\alpha}\right)_{\text {inf }}<y(\alpha)<\left(c_{\alpha}\right)_{\text {sup }}$ (Regime 1 ), then by the implicit function theorem, we have $y^{\prime}(\alpha)=-\frac{E\left[\tilde{x} U_{c y}\right]}{k v^{\prime \prime}+E\left[U_{c c}\right.}$, and $W_{\alpha \alpha}(\alpha)=E\left[\tilde{x}^{2} U_{c c}\right]+$ $y^{\prime}(\alpha) E\left[\tilde{x} U_{c y}\right]$, where all functions are taken at $y=y(\alpha)$ and $\tilde{c}_{\alpha} \cdot W_{\alpha \alpha}(\alpha)$ is negative if

$$
\frac{k v^{\prime \prime} E\left[\tilde{x}^{2} u^{\prime \prime}\right]+\eta^{2}\left(E\left[u^{\prime \prime}\right] E\left[\tilde{x}^{2} u^{\prime \prime}\right]-E\left[\tilde{x} u^{\prime \prime}\right]^{2}\right)}{k v^{\prime \prime}+\eta^{2} E\left[u^{\prime \prime}\right]}<0
$$


where the derivatives of $v($ resp. $u)$ are taken at $(1-\eta) y(\alpha)\left(\operatorname{resp} . \tilde{c}_{\alpha}-\eta y(\alpha)\right)$ and this inequality is satisfied due to the concavity of $u$ and $v$ and the Cauchy-Schwarz inequality.

When $y(\alpha)=\left(c_{\alpha}\right)_{\text {sup }}\left(\right.$ Regime 2), $W_{\alpha \alpha}(\alpha)$ is given by $W_{\alpha \alpha}(\alpha)=x_{\text {sup }}^{2}\left[k v^{\prime \prime}+E U_{y y}\right]+$ $2 x_{\mathrm{sup}} E\left[\tilde{x} U_{c y}\right]+E\left[\tilde{x}^{2} U_{c c}\right]<0$, where all functions are taken at $y=\left(c_{\alpha}\right)_{\text {sup }}$ and $c=z+\alpha \tilde{x}$. The concavity condition is then given by $k(1-\eta)^{2} u^{\prime \prime}((1-$ $\left.\eta)\left(\left(c_{\alpha}\right)_{\text {sup }}\right)\right) x_{\text {sup }}^{2}+E\left(\tilde{x}-\eta x_{\text {sup }}\right)^{2} u^{\prime \prime}\left(z(1-\eta)+\alpha\left(\tilde{x}-\eta x_{\text {sup }}\right)\right)<0$, which is automatically satisfied by concavity of $u$. The same applies for $y(\alpha)=\left(c_{\alpha}\right)_{\mathrm{inf}}$ (Regime 3).

Finally, note that $y(\alpha)$ is continuous and so is $E^{Q}\left[F_{y}\left(\tilde{c}_{\alpha}, y(\alpha)\right)\right]$. This means that when we switch from Regime 2 to Regime 1 (or from Regime 3 to Regime 1) and conversely, at some $\widehat{\alpha}>0$, we have $E^{Q}\left[F_{y}\left(\tilde{c}_{\widehat{\alpha}}, y(\widehat{\alpha})\right)\right]=0$ and $W^{\prime}\left(\widehat{\alpha}^{-}\right)=W^{\prime}\left(\widehat{\alpha}^{+}\right)=E\left[U_{c}\left(\tilde{c}_{\widehat{\alpha}}, y(\widehat{\alpha})\right)\right]$. Thus, $W_{\alpha}(\alpha)$ is continuous at $\widehat{\alpha}$ and since $W$ is concave at the left and at the right of $\widehat{\alpha}$, it is concave on a neighborhood of $\widehat{\alpha}$. The unique remaining cases correspond to switches from Regime 2 to Regime 3 and conversely. Since $y(\alpha)$ is continuous, such a switch can only occur for $\alpha=0$. In conclusion, $W$ is concave on $\mathbb{R}_{-}$and on $\mathbb{R}_{+}$but might not be concave at 0 .

Let us then consider separately the two following problems $\max _{\alpha \geq 0,\left(c_{\alpha}\right)_{\text {inf }} \leq y \leq\left(c_{\alpha}\right)_{\text {sup }}} k \ln ((1-$

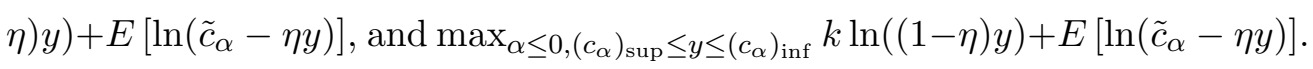

Let us start with the first one, i.e. $\alpha \geq 0$. The objective function is concave in $(\alpha, y)$ and the domain $\left\{(\alpha, y): \alpha \geq 0,\left(c_{\alpha}\right)_{\text {inf }} \leq y \leq\left(c_{\alpha}\right)_{\sup }\right\}$ is convex. The first-order necessary and sufficient conditions for an interior solution are then given by $\frac{k}{y}-\eta E\left[\frac{1}{\tilde{c}_{\alpha}-\eta y}\right]=0$, and $E\left[\frac{\widetilde{x}}{\tilde{c}_{\alpha}-\eta y}\right]=0$. Deriving $y$ from the first equation and replacing it in the second equation we obtain $\alpha=0$ which is only optimal if $k=\eta /(1-\eta)$. Otherwise there is no interior solution. The same applies for $\alpha \leq 0$.

This means that the solutions of (2) are necessarily such that $y^{*}\left(\alpha^{*}\right)=$ $z+\alpha^{*} x_{\text {sup }}$ or $y\left(\alpha^{*}\right)=z+\alpha^{*} x_{\mathrm{inf}}$. It suffices then to solve the two following 
problems $\max _{\alpha} k \ln ((1-\eta)(z+\alpha \bar{x}))+E[\ln ((1-\eta) z+\alpha(\widetilde{x}-\eta \bar{x}))]$, with $\bar{x}=$ $x_{\text {sup }}$ or $\bar{x}=x_{\text {inf }}$, and to compare their values to determine $\alpha^{*}$. We obtain $\alpha_{1}=\frac{k(1-\eta)-\eta}{(k+1)\left(\eta x_{\mathrm{sup}}-x_{\mathrm{inf}}\right)} z$ and $\alpha_{2}=\frac{\eta-k(1-\eta)}{(k+1)\left(x^{+}-\eta x^{-}\right)} z$ and $W\left(\alpha_{0}\right)-W\left(\alpha_{1}\right)=$ $(k+\pi) \ln \left(\frac{(k+\pi)(1-\eta)}{\pi(1-\eta)+\eta}\right)-(k+1-\pi) \ln \left(\frac{(k+1-\pi)(1-\eta)}{1-\pi(1-\eta)}\right)=\Delta(\pi)$. We check that $\Delta(\pi)$ is decreasing on $[0,1]$ with $\Delta(1 / 2)=0$. Consequently, $\alpha^{*}=\alpha_{1}$ for $\pi \leq \frac{1}{2}$ and $\alpha^{*}=\alpha_{2}$ for $\pi \geq \frac{1}{2}$.

\section{References}

G. A. Akerlof and W. T. Dickens. The economic consequences of cognitive dissonance. Amer. Econom. Rev., 72:307-319, 1982.

T. G. Bali, N. Cakici, and R. F. Whitelaw. Maxing out: Stocks as lotteries and the cross-section of expected returns. J. Financial Econom., 99:427446, 2011.

D. E. Bell. Disappointment in decision making under uncertainty. Oper. Res., 33:1-27, 1985.

S. Benartzti, A. Previtero, and R. H. Thaler. Annuitization puzzles. J. Econom. Perspectives, 25(4):143-164, 2011.

M. K. Brunnermeier and J. A. Parker. Optimal expectations. Amer. Econom. Rev., 95:1092-1118, 2005.

M. K. Brunnermeier, C. Gollier, and J. A. Parker. Optimal beliefs, asset prices, and the preference for skewed returns. Amer. Econom. Rev., 97: 159-165, 2007.

A. J. Caplin and J. Leahy. Psychological expected utility theory and anticipatory feelings. Quart. J. Econom., 116(1):55-79, 2001.

G. M. Constantinides. Habit formation: a resolution of the equity premium puzzle. J. Political Econom., 98:519-543, 1990. 
P. J. Cook and C. T. Clotfelter. The peculiar scale economies of lotto. Amer. Econom. Rev., 83:634-643, 1993.

M. Friedman and L. J. Savage. The utility analysis of choices involving risk. J. Political Econom., 4:279-304, 1948.

U. Gneezy, J. A. List, and G. Wu. The uncertainty effect: When a risky prospect is valued less than its worst possible outcome. Quart. J. Econom., 121:1283-1309, 2006.

W. N. Goetzmann and A. Kumar. Equity portfolio diversification. Rev. Finance, 12:433-463, 2008.

C. Gollier and A. Muermann. Optimal choice and beliefs with ex ante savoring and ex post disappointment. Management Sci., 56:1272-1284, 2010.

F. Gul. A theory of disappointment aversion. Econometrica, 59:667-686, 1991.

A. Kumar. Who gambles in the stock market? J. Finance, 64:1889-1933, 2009.

G. Loomes and R. Sugden. Disappointment and dynamic consistency in choice under uncertainty. Rev. Econom. Stud., 53:271-282, 1986.

L. L. Lopes. Between hope and fear: The psychology of risk. Advances in Experimental Social Psychology, 20:255-95, 1987.

T. Mitton and K. Vorkink. Equilibrium underdiversification and the preference for skewness. Rev. Financial Stud., 20:1255-1288, 2007.

H. Shefrin and M. Statman. Behavioral portfolio theory. J. Financial Quant. Anal., 35:127-151, 2000. 
Table 1: Allais Paradox

\begin{tabular}{lccccc}
\hline Lottery $\{0,1,5\}$ & $\mathrm{A} 1$ & $\mathrm{~A} 2$ & $\mathrm{~B} 1$ & $\mathrm{~B} 2$ \\
$Q=\left(q_{1}, q_{2}, q_{3}\right)$ & $(0,1,0)$ & $(0.01,0.89,0.1)$ & $(0.9,0,0.1)$ & $(0.89,0.11,0)$ \\
\hline Preference & \multicolumn{2}{c}{$\mathrm{A} 1 \succ \mathrm{A} 2$} & \multicolumn{2}{c}{$\mathrm{B} 1 \succ \mathrm{B} 2$} \\
\hline$k=0.75$ & $y^{*}(Q)$ & 1 & 0.7744 & 0 & 0 \\
$k$ & $W(Q)$ & -0.1728 & -0.1790 & -0.5502 & -0.5513 \\
$k=2$ & $y^{*}(Q)$ & 1 & 1.0404 & 0.1989 & 0.2002 \\
& $W(Q)$ & -0.2963 & -0.3117 & -0.9126 & -0.9132 \\
\hline
\end{tabular}

The table presents for $k$ equal to 0.75 and 2 the choice of an agent endowed with a utility function $U(c, y)=-(1+c-y / 2)^{-3} / 3$ between the lotteries considered in the Allais paradox. The different lotteries yield 0 with probability $q_{1}, 1$ with probability $q_{2}$ and 5 with probability $q_{3}$. They differ by the values of $\left(q_{1}, q_{2}, q_{3}\right)$. The Allais paradox is explained when $A_{1} \succ A_{2}$ and $B_{1} \succ B_{2}$. This is the case when $k$ remains sufficiently close to $\eta /(1-\eta)$ which is equal to 1 in this example.
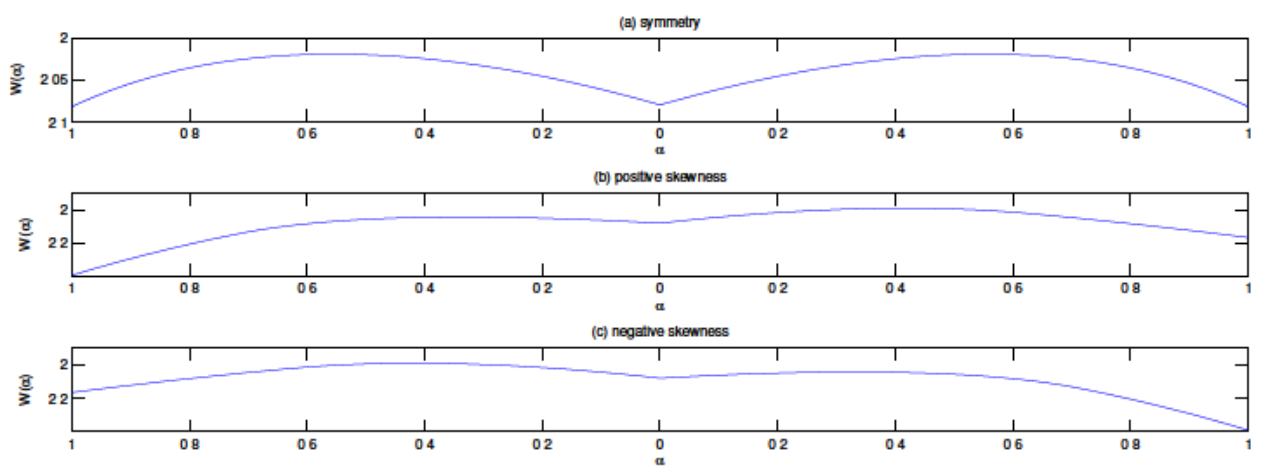

Figure 1: Skewness and welfare

This figure presents the welfare $W(\alpha)$ as a function of the investment level $\alpha$ for a symmetric (a), positively skewed (b) and negatively skewed (c) binary risk. The utility function is given by $U(c, y)=\ln (c-y / 2)$ and $k=2$. Initial wealth is given by $z=1$. (a) For $x_{\text {sup }}=-x_{\text {inf }}=0.2$ (and $\left.\pi=1 / 2\right)$, the risk is symmetric and there are two optimal investment levels $\alpha_{1}=-\alpha_{2}=0.56$. (b) If we maintain $x_{\text {inf }}=-0.2$ and take $x_{\text {sup }}$ equal to 0.4 (with $\pi=1 / 3$ to keep a zero mean), the optimal investment level is positive and given by $\alpha_{1}=0.42$. (c) If we maintain $x_{\text {sup }}=0.2$ and take $x_{\text {inf }}$ equal to -0.4 (with $\pi=2 / 3$ to keep a zero mean), the optimal investment level is negative and given by $\alpha_{2}=-0.42$. 

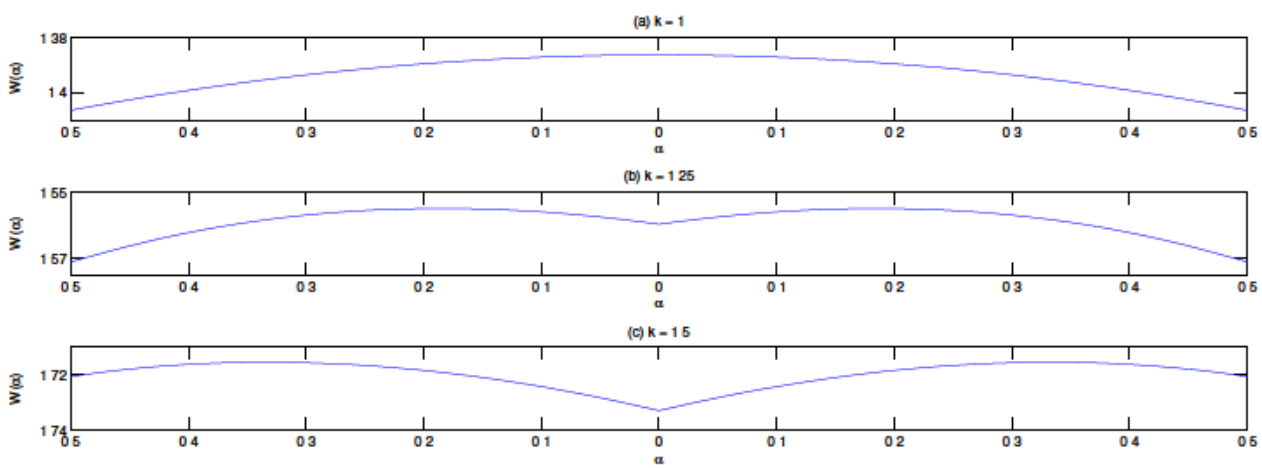

Figure 2: The intensity of anticipatory feelings and welfare

This figure presents the welfare $W(\alpha)$ as a function of the investment level $\alpha$ for three different values of the intensity of anticipatory feelings $k$ and in the case of a zero-mean symmetric binary risk $\left(x_{\mathrm{sup}}=0.2\right.$ and $\left.x_{\text {inf }}=-0.2\right)$. The utility function is given by $U(c, y)=\ln (c-y / 2)$. Initial wealth is given by $z=1$. For $k=1$, the optimal demand is zero. When $k$ increases, zero becomes a local minimum and the two (symmetric) maxima go away from zero. 